\title{
Exercise-induced ruptured renal tumour in a young patient with hereditary leiomyomata-associated renal cell carcinoma (HLRCC): a case report
}

\author{
Mohamad Fairuz Mohamad Sharin ${ }^{1}$, Mugialan Pushpanathan ${ }^{1}$, Arvind Vashdev Jagwani ${ }^{1}$ \\ Khairul Asri Mohd Ghani ${ }^{1}$, Saiful Azli Mohd Zainuddin², Noorjehan Omar ${ }^{3}$ and Firdaus Hayati ${ }^{*^{*}}$ (i)
}

\begin{abstract}
Background: Hereditary leiomyomatosis-associated renal cell carcinoma (HLRCC) is rare with only 300 families reported to date worldwide. Despite highlighting renal malignancy, it is infamous for its cutaneous and leiomyomatosis component as the more common cause of presentation. This report is to highlight the rare presentation of HLRCC which occurs in a teenager which is unfortunately complicated with exercise-induced renal tumour rupture.

Case presentation: A 22-year-old gentleman presented with severe right lower abdominal pain for 4 days, associated with fever and nausea following a session of strenuous exercise. He has first-degree relatives and second-degree relatives from his maternal side who were genetically tested positive for HLRCC. Clinical examination revealed a tender right lumbar and right iliac fossa region. A computed tomography of the kidneys demonstrated a ruptured right renal tumour with a large right renal subcapsular and contained right retroperitoneal hematoma. An emergency right radical nephrectomy was performed, and he recovered well. The histopathological examination was consistent with HLRCC associated ruptured right renal cell carcinoma.

Conclusion: Identification of this underreported familial malignancy is paramount as the onset of neoplasia occurs much earlier in life. This necessitates screening of family members at a younger age, and the management is followed by life-long surveillance.
\end{abstract}

Keywords: Exercise, Leiomyoma, Renal cell carcinoma

\section{Background}

Hereditary leiomyomatosis-associated renal cell carcinoma (HLRCC) is a rare autosomal dominant condition caused by germline mutation of gene encoding fumarate hydratase (FH) with only 300 families that have been reported worldwide thus far [1]. Patients usually present at a mean age of 25 and they more commonly come

\footnotetext{
*Correspondence: m_firdaus@ums.edu.my; firdaushayati@gmail.com

${ }^{4}$ Department of Surgery, Faculty of Medicine and Health Sciences,

Universiti Malaysia Sabah, Kota Kinabalu, Sabah, Malaysia

Full list of author information is available at the end of the article
}

with cutaneous leiomyomas (76-90\%), uterine fibroids in affected females (80-90\%) and rarely renal cell carcinoma (15-20\%) [1]. FH gene defect is reported to be the genetic basis and underscores the importance of dermatologic and genetic diagnosis of HLRCC so that appropriate routine cancer screening and counselling of the patient and relatives at risk can be instituted, especially for early tumour detection. Ruptured HLRCC, especially induced by exercise, has never been reported to date. We report a case of HLRCC, diagnosed prior to genetic screening and later presented with exercise-induced rupture of renal cell carcinoma. 


\section{Case presentation}

A 22-year-old gentleman presented to the casualty with severe right lower abdominal pain radiating to the back for 4 days, associated with fever and nausea. He was previously well. Symptoms began after a session of noncontact strenuous exercise as he was involved in noequipment workout. He denied episodes of haematuria or passing out blood clots. On presentation, the patient remained hemodynamically stable with a tender and guarded right lumbar and right iliac fossa region. There were no obvious skin lesions suggestive of cutaneous leiomyomas found.

Further history revealed that the patient's first-degree and second-degree relatives from his maternal side were genetically tested positive for HLRCC 5 years ago after his aunt was found to have renal cell carcinoma at the age of 16. (The patient was 17-year old then.) Before the genetic diagnosis, 3 family members passed away in their 20's due to unknown causes, but all presented with acute abdominal pain before their demise. The patient was advised for routine screening with a yearly kidney magnetic resonance imaging (MRI), but he had defaulted follow-up due to personal reasons.

An urgent multiphasic computed tomography (CT) of the kidneys demonstrated a ruptured right renal tumour with a large right renal subcapsular and contained right retroperitoneal hematoma (Figs. 1 and 2). There was no evidence of uterine leiomyomas and distant metastasis in the CT images. He was immediately resuscitated and planned for surgery. We performed an emergency right radical nephrectomy via a subcostal incision, and noted intraoperatively, the right kidney was grossly enlarged due to subcapsular hematoma (Fig. 3). Due to dense adhesion, the renal capsule had to be divided to identify the renal hilar vessels for safe ligation. Two renal arteries and a renal vein were ligated successfully with hemo-lock; then, the ureter ligated and divided. Organized clots were evacuated. Postoperatively, the patient recovered well. Histopathological examination of the resected right kidney shows a lower pole subcapsular tumour with superficial renal cortex infiltration and capsular rupture with exophytic growth. It was reported as HLRCC associated ruptured right renal cell carcinoma (pT4). The patient recovered well, discharged on postoperative day 7 , and will be under close surveillance afterwards.

\section{Discussion}

Non-traumatic renal haemorrhage into the subcapsular and perinephric space is rare especially if it occurs after exercise. This condition is also known as Wunderlich's syndrome, which can be caused by renal neoplasms including HLRCC (61.2\%), idiopathic causes (38\%),
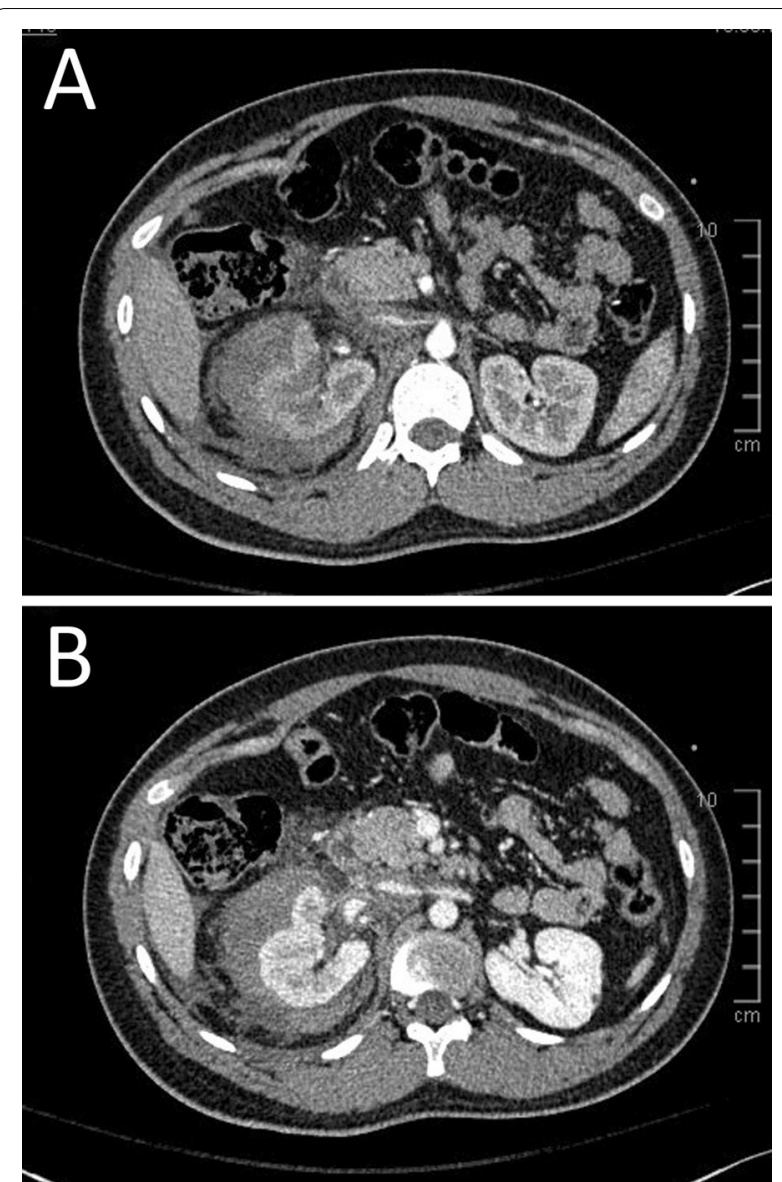

Fig. 1 A An axial cut of contrast-enhanced CT of the kidney at (B) cortical-medullary phase: a right-sided upper pole renal tumour and at $\mathbf{B}$ nephrographic phase: an enhancing right-sided upper pole renal tumour

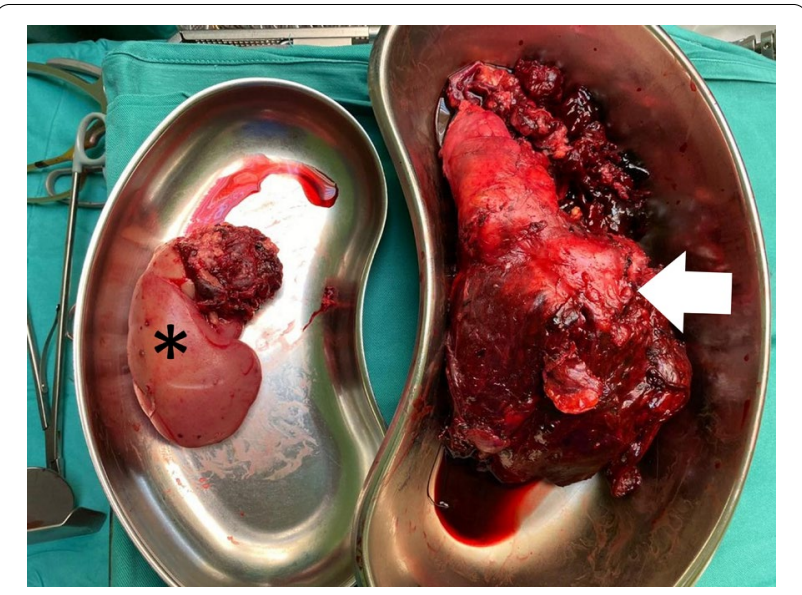

Fig. 2 Right kidney specimen with an upper pole tumour $\left(^{*}\right)$. Peri-renal fat tissues were seen with the surrounding hematoma (arrow) 

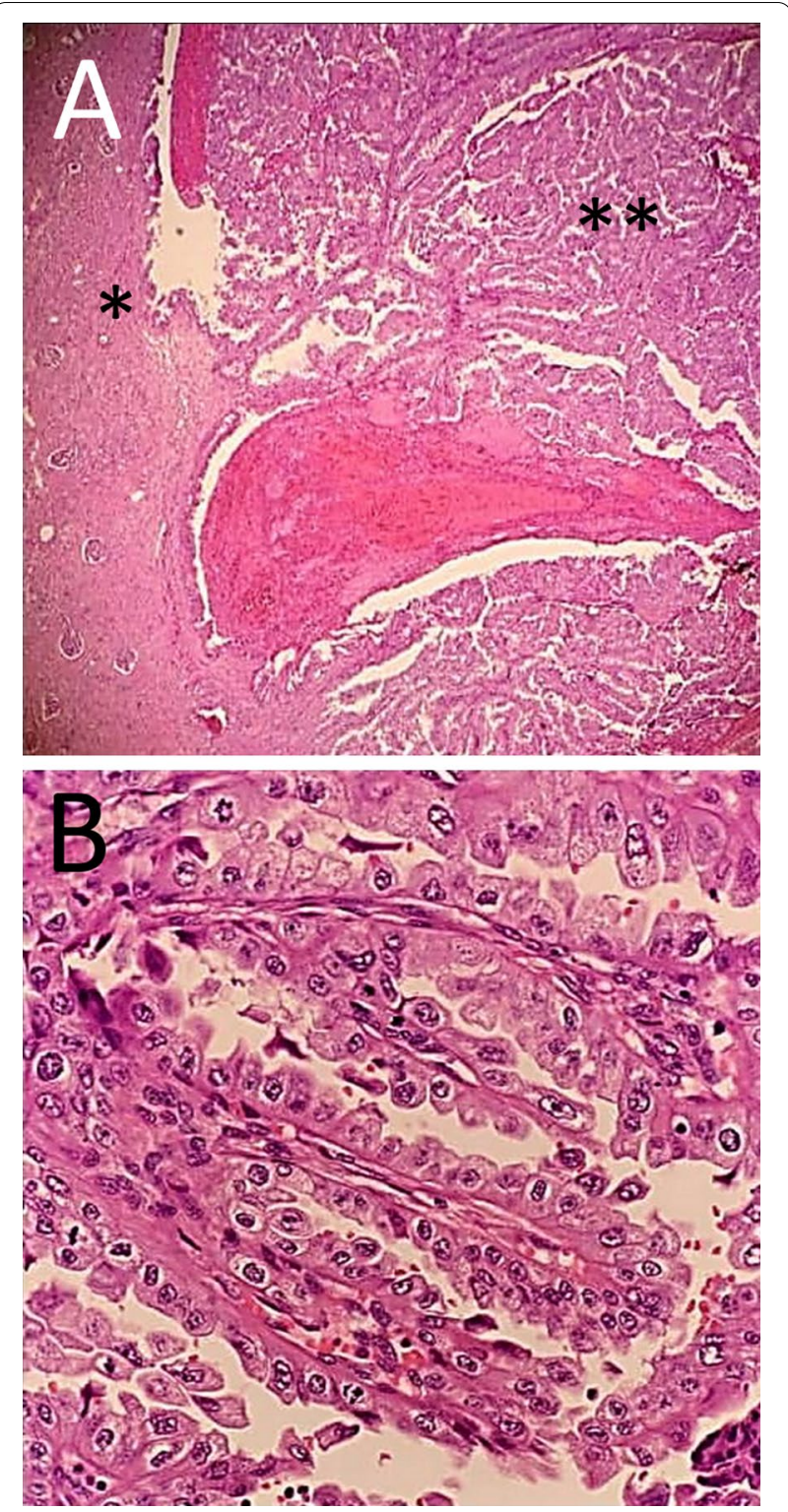

Fig. 3 Right kidney tumour; $\mathbf{A}$ tumour ${ }^{* *}$ ) with papillary architecture arising from the renal cortex $\left.{ }^{*}\right)$ and rupturing the capsule $(H \& E, \times 40)$ and $\mathbf{B}$ the tumour papillae are lined by large cells with abundant eosinophilic cytoplasm having large vesicular nuclei with prominent inclusion like eosinophilic nucleoli and perinuclear clearing $\left(H \& E_{1} \times 400\right)$
The mechanism by which $\mathrm{FH}$ acts as a tumour suppressor gene is still unknown; however, there are hypotheses that state hypoxia-induced metabolic pathway is involved in oncogenesis [3]. It is a very rare disorder, affecting male and females equally but postulated to be underreported due to its rarity and lack of awareness among clinicians [4]. This patient and his family, however, have been successfully diagnosed much earlier but unfortunately defaulted follow-up and presented with this uncommon complication.

HLRCC is identified by three main characteristics, namely (a) multiple cutaneous piloleiomyomas, (b) multiple early onset uterine leiomyomas among females, and (c) an early onset form of type 2 papillary renal cell cancer. Our patient belongs to the group absent of cutaneous manifestations, instead had a renal tumour. Due to the risk of developing an aggressive form of renal cancer, early genetic testing by predictive $\mathrm{FH}$ mutation beginning at the age of 16 to 18 years old and in confirmed cases of HLRCC, annual kidney MRI has been recommended [4]. Up to date, there is no available tumour marker for screening of HLRCC [4].

Treatment should be initiated as a life-saving procedure by giving fluid resuscitation, blood transfusion and reversal of coagulopathy to ensure optimized bodily functions. For patients in shock or ongoing bleeding, immediate treatment is mandatory by selective arterial embolization and/or operative management such as nephrectomy [5]. The earlier treatment can be performed aiming for preservation of functional renal parenchyma in the presence of interventional radiological expertise. In contrast to embolization, surgery as in our case has the important advantage of allowing for pathologic evaluation for a definitive diagnosis.

Although multiple previous cases have been reported regarding this rare disease, our patient's presentation demonstrated an aggressive form of renal malignancy, a less common manifestation of HLRCC. Even though he did not have distant metastasis, a younger age of presentation and the complication of tumour rupture is likely a poor prognostic factor. This is the first such case being reported from the South East Asian population, and the first-ever reported case of HLRCC with exercise-induced tumour rupture.

\section{Conclusion}

HLRCC is a rare form of genetically predisposed renal malignancy that should be detected early when there is suspicious genealogy or pathognomonic clinical manifestations. Clinicians should be vigilant in genetically establishing the diagnosis, screen family members at risk and advice for close surveillance. Patients must be aware of its 
complications including the effect after slight non-contact strenuous exercises.

\section{Abbreviations}

CT: Computed tomography; FH: Fumarate hydratase; HLRCC: Hereditary leiomyomata-associated renal cell carcinoma; MRI: Magnetic resonance imaging.

\section{Acknowledgements}

We acknowledge those who have been directly or indirectly involved in the management of this patient throughout his hospitalization and discharge.

\section{Authors' contributions}

MP and AVJ wrote the initial manuscript. MFMS performed the literature review. SAMZ and NO provided the study material. KAMG supervised the manuscript writing. FH revised the manuscript and became the corresponding author. All authors read and approved the final manuscript.

\section{Funding}

Not applicable.

\section{Availability of data and materials}

The dataset used and/or analyzed during the current study is available from the corresponding author on reasonable request.

\section{Declarations}

\section{Ethics approval and consent to participate}

Ethical approval was not required as this is a case report. Informed written consent to participate was provided by all participants.

\section{Consent for publication}

Written consent was obtained from the patient for publication of this case report and accompanying image.

\section{Competing interests}

The authors declare they have no competing interests.

\section{Author details}

${ }^{1}$ Department of Urology, Faculty of Medicine and Health Sciences, Universiti Putra Malaysia, Seri Kembangan, Selangor, Malaysia. ${ }^{2}$ Department of Urology, Serdang Hospital, Kajang, Selangor, Malaysia. ${ }^{3}$ Department of Pathology, Serdang Hospital, Kajang, Selangor, Malaysia. ${ }^{4}$ Department of Surgery, Faculty of Medicine and Health Sciences, Universiti Malaysia Sabah, Kota Kinabalu, Sabah, Malaysia.

Received: 25 August 2021 Accepted: 25 November 2021

Published online: 11 December 2021

\section{References}

1. Alam NA, Barclay E, Rowan AJ et al (2005) Clinical features of multiple cutaneous and uterine leiomyomatosis: an underdiagnosed tumor syndrome. Arch Dermatol 141(2):199-206

2. Zhang JQ, Fielding JR, Zou KH (2002) Etiology of spontaneous perirenal hemorrhage: a meta-analysis. J Urol 167:1593-1596

3. Pollard P, Wortham N, Barclay E et al (2005) Evidence of increased microvessel density and activation of the hypoxia pathway in tumours from the hereditary leiomyomatosis and renal cell cancer syndrome. J Pathol 205(1):41-49

4. Menko FH, Maher ER, Schmidt LS et al (2014) Hereditary leiomyomatosis and renal cell cancer (HLRCC): renal cancer risk, surveillance and treatment. Fam Cancer 13(4):637-644

5. Hayati F, Zainal Abidin ZA, Kadir F, Nik Ismail NA, Tan GH (2017) Embolization in haemorrhage-associated transurethral resection of prostate: an advancement of endovascular technique. Medeni Med J 32(2):123-127

\section{Publisher's Note}

Springer Nature remains neutral with regard to jurisdictional claims in published maps and institutional affiliations.

\section{Submit your manuscript to a SpringerOpen ${ }^{\circ}$ journal and benefit from:}

- Convenient online submission

- Rigorous peer review

- Open access: articles freely available online

- High visibility within the field

- Retaining the copyright to your article

Submit your next manuscript at $\boldsymbol{\nabla}$ springeropen.com 